\title{
Issue Thérapeutique Des Patients Tuberculeux Pulmonaires Biologiquement Confirmés Dans La Zone Sanitaire De Parakou-N’Dali De 2011 À 2015
}

\author{
CA Dovonou \\ CA Alassani \\ $S$ Ade \\ CA Attinsounon \\ S Ahoui \\ FT Tognon
}

Service de Médecine, Centre Hospitalier Universitaire du Borgou, Parakou. Département de Médecine et Spécialités Médicales, Faculté de Médecine, Université de Parakou

\section{DM Zannou \\ G Ade \\ F Houngbe}

Département de Médecine et Spécialités Médicales, Faculté des Sciences de la Santé de Cotonou, Université d’Abomey-Calavi

doi: 10.19044/esj.2016.v12n33p340 URL:http://dx.doi.org/10.19044/esj.2016.v12n33p340

\begin{abstract}
Objective: The objective of this study is to describe the therapeutic outcome and factors associated with treatment failure in laboratory confirmed pulmonary tuberculosis patients.

Methods: He acted in a descriptive and analytical study referred to prospective data collection. The study population consisted of laboratory confirmed pulmonary tuberculosis patients in the health zone Parakou-N'Dali from 2011 to 2015.

Results: A total of 313 TB patients were enrolled. The average age of patients was $37.4 \pm 14.3$ years. The sex ratio was 2.1 . Two hundred sixtyfive (265) patients were cured, a cure rate of $84.66 \%$; 5 patients had completed treatment. Therapeutic success was observed in 270 patients (86.26\%); the rate of treatment failure was 2.24\%. Twenty-nine (29) patients died (9.26\%); 6 patients were lost to follow and only 1 was transferred. Factors associated with treatment failure were the BMI screening to the lower than $18.5 \mathrm{~kg} / \mathrm{m}^{2}(\mathrm{p}=0.000)$ and hospitalization $(\mathrm{p}=0.002)$.
\end{abstract}


Conclusion: The therapeutic outcome of patients with pulmonary tuberculosis in the health zone Parakou-N'Dali was characterized by a low rate process complete, lost sight and transfers.

Keywords: Therapeutic outcome, pulmonary tuberculosis, Benin

\section{Résumé}

Objectif: L'objectif de cette étude était de décrire l'issue thérapeutique et les facteurs associés à l'échec thérapeutique chez les patients tuberculeux pulmonaires biologiquement confirmés.

Méthodes: Il s'est agi d'une étude descriptive et visée analytique, à collecte de données prospective. La population d'étude était constituée des patients tuberculeux pulmonaires biologiquement confirmés dans la zone sanitaire Parakou-N'Dali de 2011 à 2015.

Résultats: Au total, 313 patients tuberculeux ont été inclus. L’âge moyen des patients était $37,4 \pm 14,3$ ans. Le sex-ratio était de 2,1. Deux cent soixantecinq (265) patients étaient guéris, soit un taux de guérison de 84,66\%; 5 patients avaient terminé leur traitement. Le succès thérapeutique était observé chez 270 patients (86,26\%); le taux d'échec thérapeutique était de 2,24\%. Vingt et neuf (29) patients étaient décédés (9,26\%); 6 patients étaient perdus de vue et 1 seul a été transféré. Les facteurs associés à l'échec thérapeutique étaient l'indice de masse corporelle au dépistage inférieur à $18,5 \mathrm{~kg} / \mathrm{m}^{2}(\mathrm{p}=0,000)$ et l'hospitalisation $(\mathrm{p}=0,002)$.

Conclusion: L'issue thérapeutique des patients atteints de la tuberculose pulmonaire dans la zone sanitaire Parakou-N’Dali était caractérisée par un faible taux de traitement terminé, de perdus de vue et de transferts.

Mots clés: Issue thérapeutique, tuberculose pulmonaire, Bénin

\section{Introduction}

La tuberculose demeure un problème de santé publique dans le monde (Gadoev et al, 2015). En 2013, environ 9 millions de personnes étaient atteintes de la tuberculose dont 1,5 million de décès (García-Basteiro et al, 2016). Les pays en développement sont les plus touchés. En effet, $56 \%$ des patients tuberculeux se retrouvent dans ces pays dont $29 \%$ en Afrique au sud du Sahara (Gebrezgabiher et al, 2016). L'Afrique reste le continent le plus touché avec 255 cas/100000 habitants soit plus du double de l'incidence mondiale (Attinsounon et al, 2014). Le Bénin n'est pas épargné par cette pandémie. En 2014, parmi les 3977 cas de tuberculose toutes formes confondues, 90,6\% avaient une tuberculose pulmonaire biologiquement confirmée. L'avènement de la chimiothérapie antituberculeuse efficace a amélioré considérablement le pronostic des patients. Au Bénin, pour une 
meilleure efficacité du traitement, celle-ci est gratuite et est directement observée. Malgré l'efficacité du traitement antituberculeux et les dispositions prises par l'Etat béninois, il n'est pas rare d’observer des cas d'échec thérapeutique. De plus, vu le traitement au long cours de la tuberculose, des cas d'abandon du traitement ou de pertes de vue sont fréquemment observés. Ceci peut contribuer au développement des mycobactéries résistantes aux antituberculeux et une propagation de la maladie; cela pourrait compromettre l'atteinte de l'objectif du traitement de la tuberculose qui est de traiter avec succès au moins $85 \%$ des patients dépistés. La présente étude avait pour objectif de décrire l'issue thérapeutique des patients atteints de tuberculose pulmonaire biologiquement confirmée suivis dans la zone sanitaire ParakouN'Dali et d'identifier les facteurs associés à l'échec thérapeutique. Les résultats de l'étude pourraient contribuer à l'amélioration de l'efficacité du Programme National de Lutte contre la Tuberculose.

\section{Patients et méthodes}

Il s'est agi d'une étude descriptive à visée analytique à collecte de données prospective qui a duré 5 ans (du 1 $1^{\mathrm{er}}$ janvier 2011 au 31 décembre 2015). La population d'étude était constituée des patients atteints de tuberculose pulmonaire biologiquement confirmée, suivis dans la zone sanitaire Parakou-N'Dali. Les enfants de moins de 15 ans ont été exclus de l'étude. La confirmation de la tuberculose pulmonaire a été faite par la recherche du bacille de Koch dans les crachats. L’issue thérapeutique a été appréciée à travers la guérison, le traitement terminé, l'échec thérapeutique, les perdus de vue, le transfert et le décès.

Les patients étaient considérés comme guéris lorsqu’ils ont présenté des résultats négatifs à l'examen des frottis au cours du dernier mois de traitement ou au moins une fois auparavant. Le traitement était terminé lorsque le patient tuberculeux a terminé le traitement sans signe d'échec mais il n’existe pas de données indiquant que les résultats de l'examen des frottis ont été négatifs au cours du dernier mois de traitement et au moins une fois auparavant ; soit parce que les tests n’ont pas été réalisés, soit parce que les résultats ne sont pas disponibles. Les patients ayant un succès thérapeutique sont ceux guéris ou ayant terminé le traitement. L’échec thérapeutique était confirmé lorsque le patient présente des résultats positifs selon l'examen des frottis après cinq mois de traitement ou plus. Le patient tuberculeux est considéré comme perdu de vue lorsqu'il n’avait pas entamé le traitement antituberculeux ou celui-ci a été interrompu pendant deux mois consécutifs ou plus. Les patients étaient considérés comme des transférés lorsqu’ils étaient transférés dans un autre centre de prise en charge quelle que soit la raison. Les décès considérés étaient ceux enregistrés chez les patients tuberculeux au cours du traitement ou avant de l'avoir commencé. Les 
patients ont été suivis pendant 6 mois pour les nouveaux cas et 8 mois pour les anciens cas conformément à la durée du traitement prévu par le protocole national. L’indice de masse corporelle (IMC) a été calculé en faisant le rapport du poids en kg par la taille en mètre au carré. Un IMC inférieur à $18,5 \mathrm{~kg} / \mathrm{m}^{2}$ traduit la dénutrition. Les données ont été codifiées, saisies à l'aide logiciel Epi-data 3.1 et Excel ensuite analysées avec les logiciels Epi info7. Les associations entre les variables étaient déterminées à l'aide du test de $\mathrm{chi}^{2}$ ou du test exact de FISCHER. Un seuil de significativité inférieur à 5\% a été considéré.

\section{Résultats}

Au total, 313 patients tuberculeux ont été inclus. L’âge moyen des patients était $37,4 \pm 14,3$ ans. La tranche d’âge de 25-34 ans était la plus touchée (31,0 \%). Le sex-ratio est de 2,1. La majorité des patients (94,25 \%) était des nouveaux cas. Cinquante et six (56) patients (17,89\%) étaient coinfectés par le VIH. La plupart des patients (83,38 \%) étaient dénutris $(n=261)$. Le tabagisme et l'alcoolisme étaient observés respectivement chez $66(21,09 \%)$ et 96 patients (30,7\%). Un (1) patient sur quatre (4) avait été hospitalisé $(24,2 \%, n=76)$. Ces différents résultats sont présentés dans le tableau I.

Une prise de poids progressive était observée chez les patients sous traitement antituberculeux (Tableau II). Le gain de poids moyen pendant les 6 mois de traitement observé était de 5,7 kilogrammes.

Deux cent soixante-cinq (265) patients étaient guéris, soit un taux de guérison de 84,66\%; 5 patients ont terminé leur traitement. Le succès thérapeutique était observé chez 270 patients $(86,26 \%)$, le taux d'échec thérapeutique était de 2,24 \% (n=7). Vingt et neuf (29) patients $(9,26 \%)$ étaient décédés, 6 patients étaient perdus de vue (1,92\%) et 1 seul avait été transféré $(0,32 \%)$. Ces différents résultats figurent dans le tableau III.

Les facteurs associés à l'échec thérapeutique étaient l’IMC au dépistage inférieur à $18,5 \mathrm{~kg} / \mathrm{m}^{2}(\mathrm{p}=0,000)$ et l'hospitalisation $(\mathrm{p}=0,002)$. Le tableau IV présente ces différents résultats.

\section{Discussion}

Cette étude à collecte prospective s’est déroulée sur 5 ans et a permis de suivre les patients atteints de tuberculose pulmonaire sur 6 mois pour les nouveaux cas et sur 8 mois pour les anciens conformément à la durée du traitement afin de décrire l'issue thérapeutique et les facteurs associés à l'échec thérapeutique. La méthodologie adoptée a permis d’apprécier l'évolution clinique et biologique des patients. La population d'étude était jeune avec une moyenne d’âge de 37,4 $\pm 14,3$ ans. Cette jeunesse de la population des patients atteints de tuberculose était aussi soulignée par 
García-Basteiro et al., (García-Basteiro et al, 2016) et Oshi (Oshi et al, 2014) avec respectivement une moyenne d'âge de 36,4 $\pm 15,7$ ans et 36,3 \pm 10,5 ans. Une prédominance masculine était observée dans la présente étude; la même tendance était rapportée par Gebrezgabiher (Gebrezgabiher et al, 2016) et de Nagpal (Nagpal et al, 2015) où les hommes représentaient en effet, respectivement $50,9 \%$ et $59,6 \%$ de leur population d'étude. Le sexe masculin peut être donc un facteur de risque de la tuberculose pulmonaire. Une conclusion ne peut pas être tirée car cette assertion ne fait pas l'unanimité. Selon Malangu et al., c’était plutôt la prédominance féminine qui était observée (Malangu et al, 2014).

La co-infection par le VIH était retrouvée chez 56 patients (17,89 \%). Oshi et al., avaient rapporté une prévalence de co-infection VIH-tuberculose de 20.5\% dans leur série (Oshi et al, 2014).

L’issue thérapeutique des patients de notre série était caractérisée par un taux de guérison de $84,66 \% ; 1,6 \%$ des patients avaient terminé leur traitement soit un taux de succès thérapeutique de 86,26\%. Le taux d'échec thérapeutique était de 2,24 \%. Le taux de décès était de 9,26\%; 1,92 \% des patients étaient perdus de vue et 1 seul avait été transféré. Le taux de guérison des patients atteints de tuberculose dans la zone sanitaire ParakouN’Dali était ainsi faible. L’objectif du programme National de lutte contre la Tuberculose (PNT) étant d'au moins $85 \%$ de taux de guérison, des efforts restent à faire pour améliorer ce taux. Un dépistage précoce et une bonne observance du traitement pourront améliorer le taux de guérison. D’autres auteurs avaient rapporté un taux de guérison similaire au nôtre. C'est le cas de Gadoev et al. (Gadoev et al, 2015) et de Nagpal et al. (Nagpal et al, 2015) qui avaient rapporté respectivement un taux de guérison de $83 \%$ et 84\% dans leurs cohortes. Des taux de guérison plus faibles ont été rapportés. En effet, Gebrezgabiher et al. (Gebrezgabiher et al, 2016) et Van Hest et al. (Van Hest et al, 2013) rapportèrent respectivement des taux de guérison de 11,8 \% et de 67,1\%. Nagpal et al. (Nagpal et al, 2015) avait observé un faible pourcentage des patients qui avaient terminé leur traitement; ce qui corrobore nos résultats. Le taux de succès thérapeutique de la présente était comparable à celui rapporté par plusieurs études [6,10]. Le taux d'échec thérapeutique dans la présente étude était faible (2,4\%). Aketi et al., (Aketi et al, 2016), García-Basteiro et al. (García-Basteiro et al, 2016) et Tweeya et al. (Tweeya et al, 2013) avaient également rapporté des taux d’échec thérapeutique similaires au nôtre avec respectivement 2,4\%, 1,1\% et 1\%. Le taux de décès était faible (9,26\%). Des taux similaires de décès ont été rapportés par Chee et al. (Chee et al, 2014) et Reis-Santos et al. (Reis-Santos et al, 2014) respectivement $10,2 \%$ et $14 \%$. Des efforts restent à fournir afin de réduire ce taux de mortalité lié à la tuberculose pulmonaire. Certains auteurs ont rapporté des taux de décès plus faibles. Aketi et al., (Aketi et al, 
2016) et Gebremariam et al. (Gebremariam et al, 2016), avaient rapporté respectivement des taux de décès de $1,4 \%$ et $1,7 \%$. A l’opposé, des taux de décès plus élevés variant entre 15 et $19 \%$ ont été rapportés par GarcíaBasteiro et al. (García-Basteiro et al, 2016) et Oshi et al (Oshi et al, 2014).

Très peu de patients (1,92\%) sont perdus de vue. Ce pourcentage est faible mais des efforts restent à faire pour tendre vers l'idéal de $0 \%$ de perdu de vue. Gebreegziabher et al. (Gebrezgabiher et al, 2016) et Tweya et al. (Tweeya et al, 2013) avaient rapporté des taux de perdus de vue similaires au nôtre, respectivement $1,5 \%$ et $1 \%$. Par rapport aux perdus de vue, la situation était moins préoccupante dans la zone sanitaire Parakou-N’Dali en comparant aux résultats rapportés par Attinsounon et al. (Attinsounon et al, 2014) et García-Basteiro et al. (García-Basteiro et al, 2016) qui notèrent des taux de perdus de vue respectivement de $15,2 \%$ et $10 \%$. Seul 1 patient a été transféré à Cotonou dans le centre de référence de prise en charge des patients tuberculeux. La tuberculose peut être prise en charge dans n'importe quelle région à condition que les antituberculeux soient disponibles. Oshi et al. (Oshi et al, 2014) et Tweya et al. (Tweeya et al, 2013) rapportèrent également un faible taux de transfert.

Les facteurs associés à l'échec thérapeutique étaient un IMC au dépistage inférieur à 18,5 kg/m² et l'hospitalisation. La dénutrition favorise une dépression de l'immunité, un risque accru de développer les infections et une inefficacité du traitement médicamenteux (Calon et al, 2014 ; de Luis et al, 2014). Ceci explique l'association entre la dénutrition et l'échec thérapeutique. L'association entre l'hospitalisation et l'échec thérapeutique était également soulignée par Bloss et al. (Bloss et al, 2012). Les facteurs tels que l'âge, le sexe, l'infection VIH, le type d'infection, le tabagisme, l'alcoolisme, l'association du traitement à l'indigénat, le niveau d'instruction et la provenance n’étaient pas associés à l'échec thérapeutique. Malangu et al. (Malangu et al, 2014) n’ont trouvé aucune association entre l'échec thérapeutique et l’âge, le sexe et le traitement à l'indigénat. Dans l'étude de Oshi et al. (Oshi et al, 2014), aucune association n'était trouvée entre l'échec thérapeutique, le type d'infection et le traitement antirétroviral. Aketi et al. (Aketi et al, 2016) n’ont rapporté aucune association entre l'échec thérapeutique et l'infection VIH. Le lieu de résidence n’était pas associé à l'échec thérapeutique dans l'étude de Gebremariam et al. (Gebremariam et al, 2016) et de Nagpal et al. (Nagpal et al, 2015). Dans l'étude de Villegas et al. (Villegas et al, 2016), aucune association entre l’échec thérapeutique et l'alcoolisme, le tabagisme et le niveau socio-économique n’a été trouvée.

\section{Conclusion}

L'issue thérapeutique des patients atteints de la tuberculose pulmonaire dans la zone sanitaire Parakou-N’Dali est caractérisée par un 
faible taux de traitement terminé, de perdus de vue et de transferts. Un dépistage et une prise en charge globale et précoce s’avèrent nécessaires afin de réduire le taux d’échec thérapeutique en vue d’abaisser la mortalité liée à la tuberculose et augmenter le taux de guérison.

\section{References:}

1. Gadoev J, Asadov D, Tillashaykhov M, Tayler-Smith K, Isaakidis P, Dadu A, et al. Factors Associated with Unfavorable Treatment Outcomes in New and Previously Treated TB Patients in Uzbekistan: A Five Year Countrywide Study. PLoS ONE 2015; 10(6): 1-17

2. García-Basteiro A, Respeito D, Augusto O, López-Varela E, Sacoor $\mathrm{C}$, Sequera V and al. Poor tuberculosis treatment outcomes in Southern Mozambique (2011-2012). BMC Infectious Diseases 2016; 16: 214-22

3. Gebrezgabiher G, Romha G, Ejeta E, Asebe G, Zemene E, Ameni G. Treatment Outcome of Tuberculosis Patients under Directly Observed Treatment Short Course and Factors Affecting Outcome in Southern Ethiopia: A Five-Year Retrospective Study. PLoS ONE 2016; 11(2): 1-10

4. Attinsounon CA, Fortes-Déguénonvo L, Diop SA, Cissoko $\mathrm{Y}$, Kpangon AA, Manga NM et al. Devenir des patients perdus de vue sous traitement antituberculeux à Dakar (Sénégal). Rev Pneumol Trop 2014; 21: 30-5

5. Oshi D, Oshi S, Alobu I, Ukwaja K. Profile, Outcomes, and Determinants of Unsuccessful Tuberculosis Treatment Outcomes among HIV-Infected Tuberculosis Patients in a Nigerian State. Tuberculosis Research and Treatment 2014; 1: 1-9

6. Gebreegziabher S, Yimer S, Bjune G. Tuberculosis Case Notification and Treatment Outcomes in West Gojjam Zone, Northwest Ethiopia: A Five-Year Retrospective Study. Journal of Tuberculosis Research 2016; 4: 23-33

7. Nagpal M, Devgun P, Chawla N. Performance of Directly Observed Treatment Provider affecting the treatment outcome of tuberculosis cases in Amritsar city. Ntl J Com-munity Med 2015; 7(1): 29-34

8. Malangu N, Ibrahim M. Tuberculosis treatment outcomes in patients with resistant tuberculosis at a district hospital in Kwazulu-Natal Province of South Africa. Botswana Journal of African Studies 2014; 28(1): 51-61

9. Van Hest R, Kodmon C, Verver S, Erkens C, Straetemans M, Manissero D. Tuberculosis treatment outcome monitoring in European Union countries: systematic review. Eur Respir J 2013; 41: 635-43 
10. Reis-Santos B, Gomes T, Locatelli R, de Oliveira ER, Sanchez MN, et al. Treatment Outcomes in Tuberculosis Patients with Diabetes: A Polytomous Analysis Using Brazilian Surveillance System. PLoS ONE 2014; 9(7):1-9

11. Aketi L, Kashongwe Z, Kinsiona C, Fueza S, Kokolomami J, Bolie G et al. Childhood Tuberculosis in a Sub-Saharan Tertiary Facility: Epidemiology and Factors Associated with Treatment Outcome. PLoS ONE 2016; 11(4): 1-13

12. Tweya H, Feldacker C, Phiri S, Ben-Smith A, Fenner L, et al. Comparison of Treatment Outcomes of New Smear-Positive Pulmonary Tuberculosis Patients by HIV and Antiretroviral Status in a TB/HIV Clinic, Malawi. PLoS ONE 2013; 8(2):1-6

13. Chee C, Lim L, KhinMar K, Han K, Gan S, Cutter J and al. Surveillance of tuberculosis treatment outcomes of Singapore citizens and permanent residents, 2002-2011. International Journal of Tuberculosis lung diseases 2014; 18(2):141-6

14. Gebremariam G, Asmamaw G, Hussen M, Hailemariam MZ, Asegu D, Astatkie A, et al. Impact of HIV Status on Treatment Outcome of Tuberculosis Patients Registered at Arsi Negele Health Center, Southern Ethiopia: A Six Year Retrospective Study. PLoS ONE 2016; 11(4): 1-11

15. Calon B, Ludes PO. Indications et modalités de la nutrition préopératoire chez le patient dénutri et non dénutri. Le Praticien en anesthésie réanimation. 2014; 18(1):17-25

16. de Luis DA, Culebras JM, Aller R, Eiros-Bouza JM. Surgical infection and malnutrition. Nutr Hosp. 2014; 30(3): 509-13

17. Bloss E, Chan PC, Cheng NW, Wang KF, Yang SL, Cegielski P. Increasing directly observed therapy related to improved tuberculosis treatment outcomes in Taiwan. International Journal of Tuberculosis lung diseases 2012; 16(4): 462-7

18. Villegas L, Otero L, Sterling TR, Huaman MA, Van der Stuyft P, Gotuzzo E, et al. Prevalence, Risk Factors, and Treatment Outcomes of Isoniazid- and Rifampicin- Mono-Resistant Pulmonary Tuberculosis in Lima, Peru. PLoS ONE 2016; 11(4): 1-11 
Tableau I : Caractéristiques générales des patients tuberculeux pulmonaires biologiquement confirmés suivis dans la zone sanitaire Parakou-N’Dali de 2011 à 2015 (n=313)

\begin{tabular}{|c|c|c|c|c|c|}
\hline \multicolumn{3}{|c|}{ Sexe } & $\begin{array}{c}\text { Variables } \\
\text { Tab }\end{array}$ & Tabagisme & $\%$ \\
\hline Féminin & 101 & 32,3 & Oui & 66 & 21,09 \\
\hline Masculin & 212 & 67,7 & Non & 247 & 78,91 \\
\hline \multicolumn{3}{|c|}{ Age } & \multicolumn{3}{|c|}{ Niveau d'instruction } \\
\hline$<40$ ans & 197 & 37,06 & Analphabètes & 91 & 29,07 \\
\hline$\geq 40$ ans & 116 & 62,94 & Alphabètes & 222 & 70,93 \\
\hline \multicolumn{3}{|c|}{ Catégories de patient } & \multicolumn{3}{|c|}{ Traitement à l'indigénat associé } \\
\hline Nouveaux cas & 295 & 94,25 & Oui & 19 & 6,07 \\
\hline Anciens cas & 18 & 5,75 & Non & 294 & 93,93 \\
\hline \multicolumn{3}{|c|}{ Coinfection VIH } & \multicolumn{3}{|c|}{ Provenance } \\
\hline Présente & 56 & 17,89 & Urbain & 247 & 78,91 \\
\hline Absente & 257 & 82,11 & Rural & 66 & 21,09 \\
\hline \multicolumn{3}{|c|}{ IMC au dépistage } & \multicolumn{3}{|c|}{ Densité bacillaire au dépistage } \\
\hline$<18,5 \mathrm{~kg} / \mathrm{m}^{2}$ & 261 & 83,38 & 3 croix & 104 & 33,2 \\
\hline$\geq 18,5 \mathrm{~kg} / \mathrm{m}^{2}$ & 52 & 16,62 & $<3$ croix & 209 & 66,8 \\
\hline \multicolumn{2}{|c|}{ Alcoolisme } & & \multicolumn{3}{|c|}{ Hospitalisation } \\
\hline Oui & 96 & 30,7 & Oui & 76 & 24,2 \\
\hline Non & 217 & 69,3 & Non & 237 & 75,8 \\
\hline
\end{tabular}

Tableau II : Evolution du poids moyen des patients tuberculeux pulmonaires biologiquement confirmés suivis dans la zone sanitaire Parakou-N'Dali de 2011 à 2015

Période du traitement antituberculeux

Début

Fin $2^{\mathrm{e}}$ mois

Fin $5^{\mathrm{e}}$ mois

Fin $6^{\mathrm{e}}$ mois
Moyenne pondérale

$$
(\mathrm{kg})
$$

$$
49,0 \pm 9,7
$$

$$
51,1 \pm 9,7
$$$$
53,6 \pm 9,2
$$

$54,7 \pm 9,3$

\begin{tabular}{|c|c|c|c|c|}
\hline Variables & Effectif & (n) & Pourcentage & $(\%)$ \\
\hline Guérison & & 265 & & 84,66 \\
\hline itement terminé & & 5 & & 1,60 \\
\hline ès thérapeutique & & 270 & & 86,26 \\
\hline $\begin{array}{l}\text { ec thérapeutique } \\
\text { (ET) }\end{array}$ & & 7 & & 2,24 \\
\hline Décès & & 29 & & 9,26 \\
\hline erdus de vue & & 6 & & 1,92 \\
\hline Transferts & & 1 & & 0,32 \\
\hline
\end{tabular}

Tableau III : Issue thérapeutique des patients tuberculeux pulmonaires biologiquement confirmés suivis dans la zone sanitaire Parakou-N'Dali de 2011 à 2015 (n=313) 
Tableau IV : Facteurs associés à l'échec thérapeutique chez les patients tuberculeux pulmonaires biologiquement confirmés suivis dans la zone sanitaire Parakou-N'Dali de

\begin{tabular}{|c|c|c|c|c|c|}
\hline \multicolumn{6}{|c|}{2011 à 2015} \\
\hline Variables & $\begin{array}{c}\text { Prévalence de } \\
\text { l’ET(\%) }\end{array}$ & $\mathrm{P}$ & Variables & $\begin{array}{c}\text { Prévalence de } \\
\text { l’ET(\%) }\end{array}$ & $\mathrm{p}$ \\
\hline & Sexe & & \multicolumn{3}{|c|}{$\begin{array}{l}\text { l’ET(\%) } \\
\text { Tabagisme }\end{array}$} \\
\hline Féminin & 3,0 & \multirow[t]{2}{*}{0,685} & Oui & 2,8 & \multirow{2}{*}{0,615} \\
\hline Masculin & 1,9 & & Non & 2,1 & \\
\hline & Age & & \multicolumn{3}{|c|}{ Niveau d’instruction } \\
\hline$<40$ ans & 2,9 & \multirow{2}{*}{0,530} & Analphabètes & 2,6 & \multirow[t]{2}{*}{0,386} \\
\hline$\geq 40$ ans & 2,1 & & Alphabètes & 2,2 & \\
\hline \multicolumn{3}{|c|}{ Catégories de patient } & \multicolumn{3}{|c|}{ Traitement à l'indigénat associé } \\
\hline Nouveaux cas & 2,4 & \multirow[t]{2}{*}{0,658} & Oui & 3,5 & \multirow[t]{2}{*}{0,624} \\
\hline Anciens cas & 0 & & Non & 1,8 & \\
\hline \multicolumn{3}{|c|}{ Coinfection VIH } & \multicolumn{3}{|c|}{ Provenance } \\
\hline Présente & 0 & \multirow[t]{2}{*}{0,212} & Urbain & 2,7 & \multirow[t]{2}{*}{0,632} \\
\hline Absente & 2,4 & & Rural & 2,1 & \\
\hline \multicolumn{3}{|c|}{ IMC au dépistage } & \multicolumn{3}{|c|}{ Densité bacillaire au dépistage } \\
\hline$<18,5 \mathrm{~kg} / \mathrm{m}^{2}$ & 3,1 & \multirow[t]{2}{*}{0,000} & 3 croix & 2,3 & \multirow[t]{2}{*}{0,963} \\
\hline$\geq 18,5 \mathrm{~kg} / \mathrm{m}^{2}$ & 1,9 & & $<3$ croix & 2,7 & \\
\hline \multicolumn{2}{|c|}{ Alcoolisme } & \multirow{3}{*}{0,585} & & jitalisation & \multirow{3}{*}{0,002} \\
\hline Oui & 3,3 & & Oui & 3,3 & \\
\hline Non & 2,3 & & Non & 2,2 & \\
\hline
\end{tabular}

